\title{
EFECTO DEL TIPO Y ORIENTACIÓN DE LA BOQUILLA EN LA APLICACIÓN DE PROTECTORES SOLARES EN FRUTOS DE PERA
} (Pyrus communis L.)

\section{EFFECT OF NOZZLE TYPE AND ORIENTATION ON THE APPLICATION OF PROTECTANTS IN PEAR (Pyrus communis L.)}

\author{
Sergio Behmer ${ }^{*}$, Facundo Gonzalez ${ }^{2}$, y Graciela Colavita ${ }^{2}$ \\ ${ }^{1}$ Departamento de Producción, Mecanización Agrícola, Facultad de Ciencias Agrarias, Universidad \\ Nacional del Comahue, cc 85 (8303) Cinco Saltos, Rio Negro, Argentina. \\ ${ }^{2}$ Departamento de Biología, Fisiología Vegetal, Facultad de Ciencias Agrarias, Universidad Nacional \\ del Comahue, cc 85 (8303) Cinco Saltos, Rio Negro, Argentina. \\ * Autor para correspondencia E-mail: sbehmer@yahoo.com
}

\section{RESUMEN}

Los elevados niveles de radiación solar y temperatura durante la temporada productiva, generan en los frutos de pera (Pyrus communis L.), y en otras especies, un daño fisiológico conocido como "golpe de sol", que provoca un importante descarte en fruta de exportación. Una de las técnicas de manejo sugeridas para reducir la incidencia de este tipo de daño es la aplicación de películas reflectantes sobre los frutos, mediante pulverizadores hidráulicos frutícolas con barra portaboquillas verticales. El objetivo de este estudio fue evaluar la cantidad de depósitos y el efecto termoprotector de una pantalla solar bajo distintas técnicas de aplicación. Para esto, se ensayaron boquillas abanico plano de diferentes caudales y ángulos de aplicación en contraposición con boquillas cono hueco, en un pulverizador móvil de laboratorio, sobre frutos de pera de tamaño comercial. Las boquillas abanico plano de mayor tamaño de gota, independientemente de su orientación, obtuvieron mayores depósito sobre la superficie del fruto que las boquillas de cono, alcanzando los $0,25 \mathrm{mg} \mathrm{cm}^{-2}$ requeridos para obtener una disminución de $1^{\circ} \mathrm{C}$ en la piel del fruto. Sin embargo, estas diferencias no resultaron suficientes para mejorar el efecto termoprotector obtenido por las boquillas tipo cono hueco.

Palabras clave: Pulverizadoras frutícolas, asoleado, calidad de aplicación

\section{ABSTRACT}

High radiation and temperature during the growing season causes a physiological damage in pear (Pyrus communis L.), and other fruit species, called 'sunburn', which results in important losses in exportable fruit. One of the management techniques suggested to reduce the incidence of sunburn is the application of reflective films on the fruit by using hydraulic fruit sprayers with vertical nozzle bar. The objective of this study was to evaluate the amount of deposits and the thermoprotective effect of a sun protectant under different application techniques. For this, flat-fan nozzles of different flow rates and application angles were tested against hollow cone nozzles, using a laboratory mobile bench, and spraying on pear fruit of commercial size. The flat-fan nozzles of larger droplet size, regardless of their orientation, obtained greater deposit on the fruit surface than the cone nozzles, reaching the required $0.25 \mathrm{mg} \mathrm{cm}^{-2}$ to obtain a decrease of $1^{\circ} \mathrm{C}$ in the fruit skin temperature. However, these differences were not sufficient to improve the thermoprotective effect obtained by hollow cone nozzles.

Key words: Fruit sprayers, sunburn, application quality

Recibido: 28 agosto 2019. Aceptado: 07 octubre 2019. 


\section{INTRODUCCIÓN}

La región del Alto Valle de Río Negro y Neuquén, Argentina, se caracteriza por presentar niveles de radiación solar y temperatura elevados durante los meses de verano. La presencia de estos factores climáticos genera, en frutos expuestos directamente a la radiación, un desorden fisiológico conocido como "golpe de sol". Dicha variación fisiológica en las características normales de un fruto es la causante de un 25 a un $55 \%$ del descarte en manzanas de exportación, dependiendo del cultivar (Colavita et al., 2011). El daño por sol ocurre cuando la temperatura de la piel de la fruta excede $\operatorname{los} 45^{\circ} \mathrm{C}$, aunque este valor varía según la radiación, la humedad relativa y la velocidad del viento. Además, se debe tener en cuenta que la temperatura de los frutos puede superar hasta en $18^{\circ} \mathrm{C}$ a la del aire (Racsko y Schrader, 2012).

El porcentaje de frutos que se asolean durante el ciclo de cultivo es diferente en cada temporada, y obedece a la severidad de factores determinantes como la temperatura de superficie de los frutos y la radiación solar, pero también a numerosos factores predisponentes como sensibilidad varietal, vigor de las plantas, estado fisiológico y nutricional, arquitectura de la canopea, entre otros (Alvarez et al., 2015).

La metodología de control del golpe de sol consiste en reducir la exposición de los frutos a elevadas temperatura y a la radiación solar intensa. Para ello, se han propuesto técnicas de manejo tales como métodos de poda, hidrocooling, mallas de sombreo, uso de materiales reflectantes (Raffo Benegas y Rodriguez, 2007; Chabbal et al. 2014).

La aplicación de películas de partículas reflectantes sobre los frutos y hojas ha sido propuesta como herramienta para modificar las condiciones micro ambientales del canopeo, ya que disminuye la carga térmica al limitar la cantidad de radiación incidente que puede ser absorbida por los frutos, y de este modo reducir la incidencia de golpe de sol (Glenn, 2012)

Las pantallas solares presentan algunas ventajas respecto a otras alternativas, como las mallas de sombreado o el enfriamiento por agua, ya que no requieren de inversiones en infraestructuras. Sin embargo, puede tener un impacto significativo sobre los costos de producción, debido al número de aplicaciones necesarias por temporada para reforzar la cobertura conforme el fruto va creciendo. Por ello para asegurar un uso racional de los productos, es necesario establecer su contribución relativa al descenso térmico en relación a las dosis aplicadas (Alvarez, 2010; Alvarez et al., 2015). En este sentido, el mencionado autor determinó que para obtener un descenso térmico de $1^{\circ} \mathrm{C}$ es necesario depositar un residuo efectivo sobre la cara del fruto de $0,238 \mathrm{mg} \mathrm{cm}^{-2}$ de caolín, necesitándose $0,1 \mathrm{mg} \mathrm{cm}^{-2}$ de depósitos del protector para que la temperatura del fruto disminuya $0,42^{\circ} \mathrm{C}$, resultados que dependerán del momento y calidad de la aplicación.

La utilización de pulverizadores con barra portaboquillas vertical es una técnica promisoria para la aplicación de agroquímicos en cultivos arbustivos, ya que es una manera segura y eficiente de protegerlos. Sin embargo es necesario elegir adecuadamente el tipo, tamaño y presión de las boquillas y su orientación con respecto al blanco de aplicación (Braekman et al., 2010).

Onorato y Tesouro (2006) afirmaron que las pastillas de abanico plano generan gotas de tamaños que varían entre los 100 y $500 \mu \mathrm{m}$; las de cono hueco entre 100 y $250 \mu \mathrm{m}$. Gotitas más pequeña de $150 \mu \mathrm{m}$ tienden a ser las más propensas a la deriva, ya que caen más lentamente que las gotas grandes. Nuyttens et al. (2009) afirmaron que si bien el tamaño de la gota afecta a su peso y como consecuencia de ello a su velocidad de caída, este efecto depende del tipo de boquilla. En el mismo sentido, Foqué y Nuyttiens (2011), observaron que las boquillas de abanico plano anti deriva y asistida por aire, presentan velocidades de 2,5 y 2,03 $\mathrm{m} \mathrm{s}^{-1}$, mientras que las boquillas cónicas presentaron velocidades entre 1,3 y $1,7 \mathrm{~m} \mathrm{~s}^{-1}$, a pesar de que todas las boquillas consideradas presentaban el mismo tamaño de orificio y caudal.

Turner y Matthews (2001), citados por Foqué and Nuyttiens, (2011)), utilizando boquillas cono hueco (tipo TXA) obtuvieron una buena cobertura debido a la formación de gotas finas, y una buena penetración en el follaje debido a la turbulencia de dichas gotas entre las hojas. Por ello afirman que las boquillas de cono (tipo TXA 80) resultan convenientes siempre y cuando se trabaje en condiciones no predisponentes a la deriva, ya que por su tamaño las gotas son altamente sensibles a los efectos de la deriva. Sin embargo, los mismos autores concluyeron que es posible lograr buena cobertura, incluso en el interior del follaje, empleando boquillas abanico plano anti-deriva, siempre y cuando se utilice inclinaciones de la boquilla del orden de $30^{\circ}$ hacia adelante o hacia atrás, ya que obtuvieron la misma cobertura que las boquillas de cono en el mismo sitio del follaje.

Muchos estudios afirman que la disposición de las boquillas con un cierto ángulo de inclinación con respecto al avance, resulta una técnica eficiente, fácil de ajustar y de muy bajo costo para mejorar la deposición y la penetración en el follaje; en este sentido, Foqué et al. (2014), hallaron una 
mejor deposición trabajando con un pulverizador hidráulico de barras horizontales, equipado con boquillas abanico plano y gota media, con un ángulo de inclinación de $30^{\circ}$ hacia adelante, frente a la misma boquilla sin inclinación.

Por otro lado, los tratamientos sobre el follaje desde dos direcciones mejoran los resultados de la aplicación. En este sentido, Di Prinzio et al. (2009) compararon la distribución de los depósitos de un protector solar de un pulverizador hidráulico con barra portaboquillas vertical, el prototipo presentaba cada $0,6 \mathrm{~m}$, a partir de $1,5 \mathrm{~m}$ y hasta los $4,5 \mathrm{~m}$, seis planos horizontales con dos boquillas dirigidas hacia el blanco con una orientación de $90^{\circ}$ con respecto a la dirección de avance. Sus resultados indicaron que el prototipo presentó un 20 a $45 \%$ más de depósitos, con incrementos de más del $100 \%$ en la zona superior del fruto, que un pulverizador hidroneumático de tipo divergente.

Behmer et al. (2014) construyeron un dispositivo móvil de laboratorio, simulando a un pulverizador hidráulico de barra portaboquilla vertical, con el cual evaluaron la calidad de la aplicación obtenida por diferentes arreglos sobre la barra, utilizando boquillas abanico plano. El blanco utilizado fueron tarjetas hidrosensibles adheridas a una esfera, que simulaba un fruto de tamaño natural. Sus resultados indicaron que la mejor cobertura fue obtenida por la disposición de porta boquillas de dos boquillas orientadas con un ángulo de inclinación de $30^{\circ}$, hacia adelante y hacia atrás, con respecto al blanco de aplicación, $\mathrm{y}$ equipadas con boquillas abanico plano que producen gotas de tamaño medio.
El presente trabajo tuvo como objetivo evaluar la cantidad de depósitos y el efecto termoprotector de un protector solar aplicado con un dispositivo móvil de laboratorio equipado con distintos tipos y orientación de las boquillas.

\section{MATERIALES Y MÉTODOS}

Se realizaron 5 tratamientos (Tabla 1) que difieren entre sí respecto del tipo de boquilla, la presión aplicada, la cantidad de pasadas y los ángulos de aplicación. Las variables de aplicación fueron seleccionadas de manera tal que las tasas de aplicación de todos los tratamientos fueron similares. De cada tratamiento se realizaron 3 repeticiones en forma aleatoria.

El Tratamiento 1 fue considerado como testigo por llevar el tipo de boquilla de uso más común en huertos frutales. Los tratamientos 2 y 3 presentan una sola boquilla por cada cuerpo de la barra portaboquillas, con ángulo de $90^{\circ}$ y $60^{\circ}$, respectivamente, con respecto a la dirección de avance, proponiendo una doble pasada de la aplicación para obtener la misma tasa de aplicación en todos los casos. Los tratamientos 4 y 5 presentan dos boquillas por cada tapa portaboquillas, con un ángulo de $60^{\circ}$ entre sí. En el caso del tratamiento 5 se utilizó una boquilla abanico plano de menor diámetro y a una velocidad menor evaluando la misma tasa de aplicación pero con menor tamaño de gotas.

Para cada tratamiento se utilizaron 5 frutos de pera (Pyrus communis L.) para cada una de las tres repeticiones, los que fueron colocados en una estructura metálica en forma de " $\mathrm{T}$ " sobre la

Tabla 1. Variables características de cada tratamiento: tipo y orientación de las boquillas, presión, caudal, velocidad, número de pasadas y tasa de aplicación.

Table 1. Variables of the treatments: nozzle type and orientation, pressure, flow rate, speed, number of runs per application and application rate.

\begin{tabular}{clcccccc}
\hline Tratamiento & Boquilla & $\begin{array}{c}\text { Número } \\
\text { de pasadas }\end{array}$ & Orientación & $\begin{array}{c}\text { Presión } \\
\text { (bares) }\end{array}$ & $\begin{array}{c}\text { Caudal } \\
\left(\mathbf{L ~ s}^{-1}\right)\end{array}$ & $\begin{array}{c}\text { Velocidad } \\
\left(\mathbf{m ~ s}^{-1}\right)\end{array}$ & $\begin{array}{c}\text { Ta } \\
\left(\mathbf{L ~ h a}^{-1}\right)\end{array}$ \\
\hline T1 & $\begin{array}{l}\text { Cono } \\
\text { D6/DC45 } \\
\text { Abanico }\end{array}$ & 1 & $90^{\circ}$ & 10 & 4,2 & 3,85 & 1963 \\
T2 & 2 & $90^{\circ}$ & 3 & 2,2 & 3,85 & 2070 \\
T3 & $\begin{array}{l}11006 \\
\text { Abanico }\end{array}$ & 2 & $60^{\circ}$ & 3 & 2,2 & 3,85 & 2070 \\
T4 & $\begin{array}{l}11006 \\
\text { Abanico doble }\end{array}$ & 1 & $+/-60^{\circ}$ & 3 & 4,42 & 3,85 & 2070 \\
11006 & $\begin{array}{l}\text { Abanico doble } \\
\text { T5 }\end{array}$ & 1 & $+/-60^{\circ}$ & 3 & 2,93 & 2,64 & 2000 \\
\hline
\end{tabular}

Nota: los ángulos de orientación se determinaron con respecto a la dirección de la salida de la boquilla y la dirección de avance. Ta: Tasa o volumen de aplicación $\left(\mathrm{L} \mathrm{ha}^{-1}\right)$ 
que se extendieron 5 alambres en cuyos extremos se sostuvo a cada fruto por el pedúnculo con un broche.

Se utilizó una pantalla solar a base de carbonato de calcio $\left(\mathrm{CaCO}_{3}\right)$ formulado al $25 \%$ $\mathrm{p} / \mathrm{v}\left(\right.$ Microcal $\left.^{\circledR}\right)$, bajo una concentración de 1,2\% $\mathrm{p} / \mathrm{v}$.

Se empleó un equipo de aplicación móvil de laboratorio (descripto por Behmer et al., 2014), el cual presenta un bastidor con dos barrales verticales portaboquillas coplanares de $0,6 \mathrm{~m}$ de alto, separados por 0,9 m entre sí (Fig. 1). Dicho bastidor se hizo avanzar por un riel de $6 \mathrm{~m}$ de largo, accionado mediante un motor eléctrico y poleas. El barral portaboquillas se conectó a una pulverizadora hidráulica estática accionada por la toma de potencia de un tractor.

A los efectos de incrementar la película del protector sobre los frutos, tal como se busca a campo, se realizaron dos aplicaciones de cada tratamiento, asegurando que los frutos se ubicaran en el mismo lugar y con la misma orientación en ambas aplicaciones.

La determinación de la cantidad de producto depositado por unidad de superficie sobre la cara de los frutos fue realizada mediante un método gravimétrico. Se extrajeron los restos de producto de la superficie del fruto con papel secante, previamente secado a estufa $\left(38^{\circ} \mathrm{C}\right.$ durante $48 \mathrm{~h}$ ) y pesados. Se humedecieron los mismos ligeramente en agua destilada y se limpió una porción rectangular del fruto cubierta con el protector aplicado. Una vez retirado todo el depósito de la misma, se cuantificó dicha superficie mediante un planímetro óptico (Marca Li-Cor, modelo 3100, Nebraska, EEUU). Finalmente se dejó secar nuevamente el papel con producto en estufa a $38^{\circ} \mathrm{C}$ durante $48 \mathrm{~h}$, para luego determinar por diferencia de pesadas los $\mathrm{mg}$ /superficie de producto aplicado.

Como variable explicativa se determinó la temperatura de la piel de los frutos expuestos bajo condiciones de elevada temperatura, similares a aquellas inductoras del golpe de sol o asoleado. Para ello se agruparon los frutos por repetición y se los sometió durante 90 minutos de exposición en una cámara de irradiación aislada de las corrientes de aire e iluminada por medio de reflectores halógenos R7S-15 de 500 Watts.

Las imágenes térmicas (Fig. 2) de los frutos fueron obtenidas mediante una cámara (Nikon, modelo Handy Thermo TVS-200, Tokyo, Japan). La distancia focal utilizada fue 1,0 m y la resolución espacial fue de $1,68 \mathrm{~mm}$. El valor de emisividad se ajustó en 0,95 (Hellebrand et al., 2001). Se realizó una toma cada 15 minutos

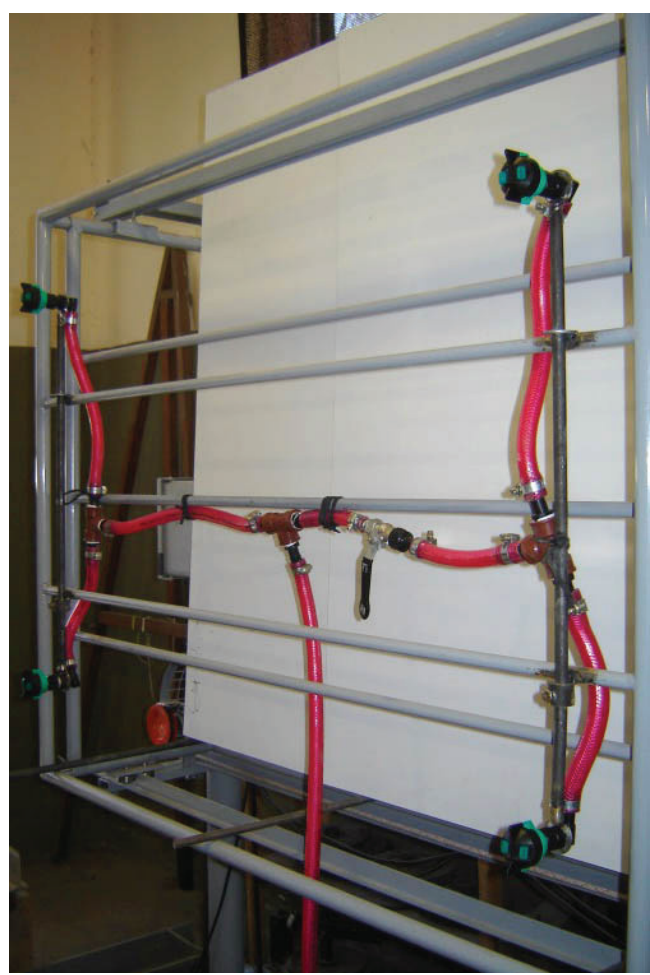

Fig. 1. Dispositivo móvil de aplicación, vista de los barrales verticales y portaboquillas.

Fig. 1. Mobile application device, view of the vertical booms and nozzle holders. 

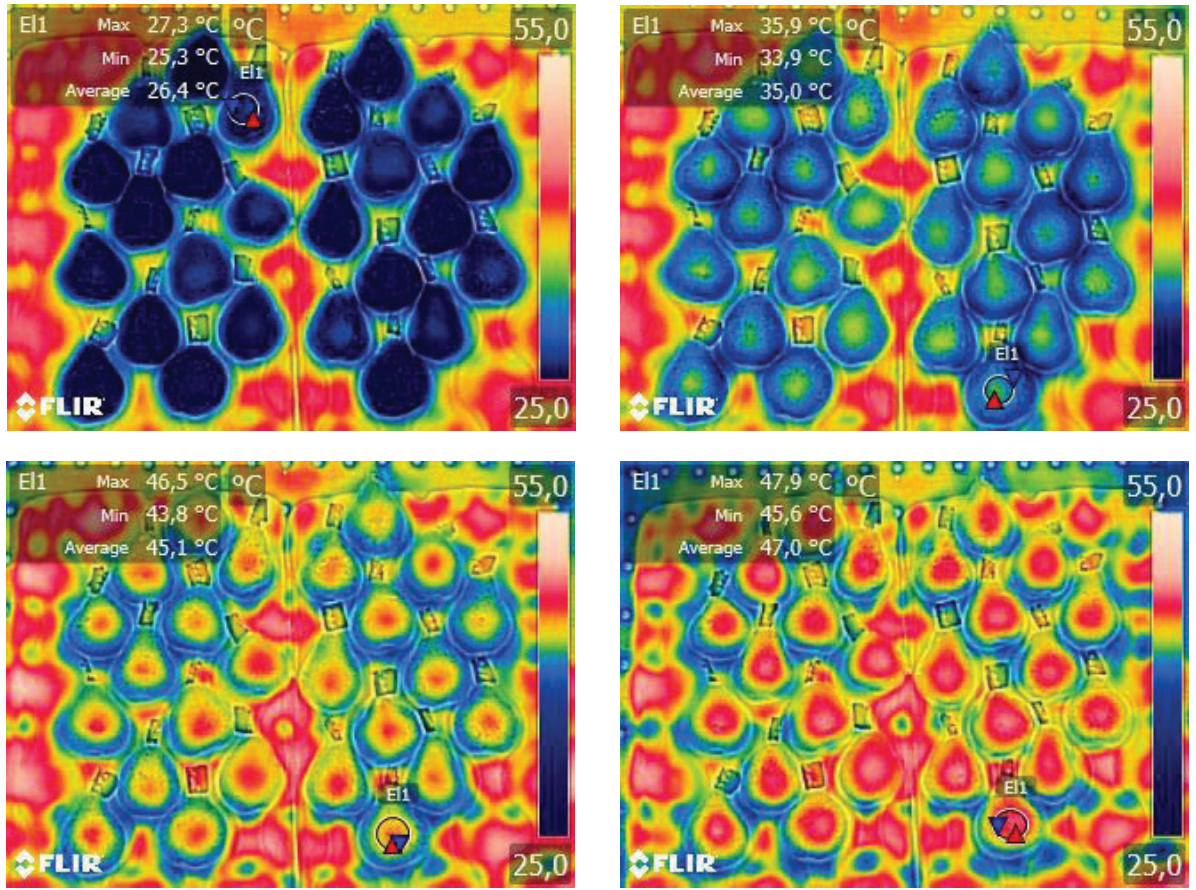

Fig. 2. Imágenes infrarroja de los frutos tratados con el protector solar.

Fig. 2. Infrared images of fruits treated with spray-on sun protectants.

durante el periodo de exposición a la lámpara mencionada. Las imágenes térmicas se analizaron con el programa para el análisis científico de imágenes (Image ${ }^{\circledR}$ 1.43u.). En cada fruta se consideró un área circular de 111 píxeles (radio aproximado $=10 \mathrm{~mm}$ ), centrado en el píxel de mayor temperatura identificado en el fruta. La temperatura promedio de cada área identificada (FST: fruit skin temperature) fue considerado como el mayor aumento térmico alcanzado por la fruta en el momento de tomar medidas. Los datos se analizaron con la subrutina ANOVA del paquete estadístico Infostat versión 2008 (Di Rienzo et al., 2008).

\section{RESULTADOS Y DISCUSIÓN}

El análisis estadístico de los depósitos por unidad de superficie hallados sobre los frutos permite afirmar que los tratamientos son diferentes entre sí, siendo aquellos realizados con la boquilla de cono D6/DC45(Tratamiento 1) y con la tapa de doble boquilla abanico plano de menor tamaño TwinCap Hypro, abanico doble 110.04, (Tratamiento 5), las que menos depósitos de producto dejaron sobre el fruto. El resto de los tratamientos no presentaron diferencias respecto de la orientación de la boquilla y la cantidad de pasadas (Tabla 2).

Estos resultados pueden explicarse con los trabajos de Nuyttiens et al. (2009) y Foqué y Nuyttens (2011), los cuales afirman que gotas pequeñas, formadas por boquillas cónicas y abanicos planos de menor diámetro, poseen bajas velocidades de salida. Adicionalmente, se debería considerar que la proyección de las gotas en los barrales portaboquillas dispuestos en posición vertical, presenta una dirección horizontal hacia el blanco de aplicación. La acción de gravedad perpendicular a la trayectoria de las gotas modificaría la dirección de las mismas alejándolas del blanco, siendo más afectadas las gotas de menor energía cinética o de menor tamaño, tales como las provenientes de las boquillas Cono D6/ DC45 y Abanico plano 10.04, TwinCap Hypro.

Los resultados obtenidos con la boquillas abanico plano inclinados con respecto a la dirección de avance, parecen contradecir las afirmaciones de Foqué y Nuyttiens (2011) y de Foqué et al. (2014), en cuanto a que el ángulo de orientación favorece la penetración en el follaje, mientras que en el presente ensayo dicha orientación no tuvo incidencia, dado que las boquillas abanico plano 11006 con diferentes propuestas de inclinación con respecto al plano de aplicación no presentaron diferencias en los depósitos hallados en los frutos (T3, T4 y T2). Sin embargo, esta discrepancia podría explicarse en la metodología utilizada en la extracción de los depósitos del fruto, ya que la misma se realizó 
Tabla 2. Depósitos del protector en el sector del fruto más expuesto a la luz solar.

Table 2. Protective deposits in the most sun-exposed fruit surface area.

\begin{tabular}{lc}
\hline Tratamientos (boquilla y orientación) & Depósitos $\mathbf{~ g ~ c m}^{-2}$ \\
\hline $5\left(\right.$ abanico doble $\left.11004,+/-60^{\circ}\right)$ & $0,13 \mathrm{~A}$ \\
$1\left(\right.$ Cono D6/DC $\left.45,90^{\circ}\right)$ & $0,13 \mathrm{~A}$ \\
$4\left(\right.$ abanico doble $\left.11006,+/-60^{\circ}\right)$ & $0,24 \mathrm{~B}$ \\
$2\left(\right.$ abanico simple $\left.11006,90^{\circ}\right)$ & $0,27 \mathrm{~B}$ \\
$3\left(\right.$ abanico simple $\left.11006,60^{\circ}\right)$ & $0,28 \mathrm{~B}$
\end{tabular}

Nota: Medias con la misma letra no son significativamente diferentes $(p>0,05)$, según Test LSD Fisher

solo en la superficie más expuesta del fruto, no evaluando la cobertura en la totalidad del fruto y por la metodología de aplicación utilizada en el estudio, ya que los frutos no estaban dentro del follaje de las plantas. A pesar de no presentar diferencia en los depósitos, se observa que el tratamiento con la tapa de doble boquilla angulada (T4) presenta ventajas competitivas respecto a los dos tratamientos de tapa simple (T2 y T3), ya que solo realizó una pasada frente al blanco con el consecuente ahorro de tiempo, combustible y presentar un menor impacto sobre el suelo.

Analizando la cantidad de depósitos obtenidos y considerando el objetivo de la aplicación de los protectores solares, se observa que sólo los tratamientos con boquillas abanico plano de mayor caudal, alcanzaron un nivel de depósitos similares a los obtenidos por Alvarez, (2010) para disminuir la temperatura de los frutos en al menos $1^{\circ} \mathrm{C}$. Hay que considerar que este resultado se logró con sólo dos aplicaciones de protector en frutos de tamaño comercial. Por lo tanto, los resultados hallados resultan promisorios, ya que en condiciones de campo las aplicaciones se realizan varias veces durante la temporada de crecimiento del fruto, por lo que es dable esperar que los depósitos obtenidos por estas boquillas en condiciones normales de campo fuesen al menos del mismo valor que los encontrados en el presente análisis.

No obstante la diferencia de depósitos hallada entre los tratamientos, no se detectaron diferencias en el efecto termoprotector entre ninguno de ellos (Fig. 3). Este resultado podría explicarse en que la diferencia entre depósitos extremos fue solo

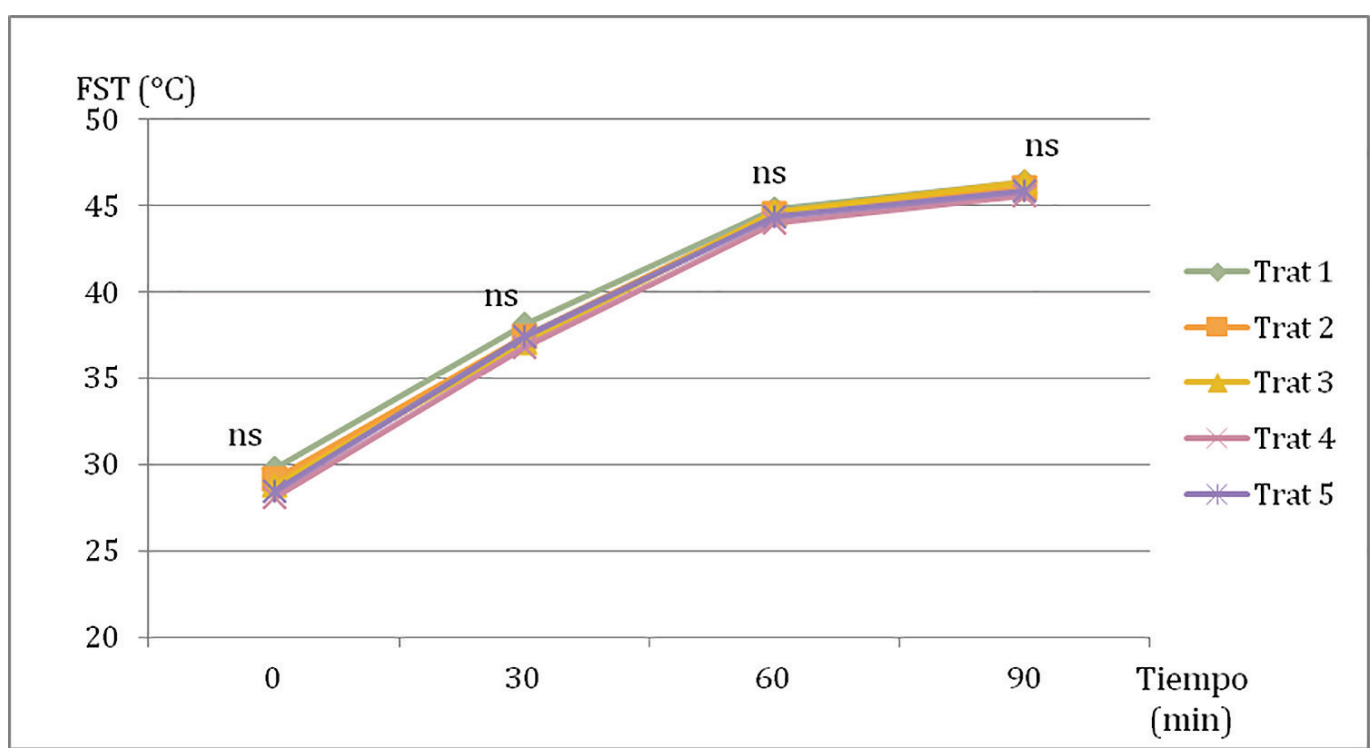

Fig. 3. Temperaturas de la piel del fruto (FST) en función del tiempo de exposición a la lámpara. Nota: $\mathrm{ns}=\sin$ diferencias según Test LSD Fisher $\alpha=0,05$

Fig. 3. Fruit skin temperatures (FST) depending on the lamp exposition time. Note: $n s=$ no differences according to Fisher LSD Test $\alpha=0.05$ 
de $0,1 \mathrm{mg} \mathrm{cm}^{-2}$, valor que permitiría esperar una disminución en la temperatura de la superficie del fruto de solo $0,5^{\circ} \mathrm{C}$, según las afirmaciones de Alvarez (2010), y esta diferencia en la temperatura no resultaría suficiente para observar diferencias significativas entre los tratamientos.

\section{CONCLUSIONES}

La cantidad de depósitos obtenida en la cara central y externa del fruto con pulverizadores hidráulicos para frutales con barral porta boquillas vertical depende del tipo de boquilla utilizada para su aplicación y es independiente de su orientación.

La mayor cantidad de depósitos obtenida por las boquillas abanico plano no fue suficiente para mejorar el efecto termoprotector obtenido por las boquillas tipo cono hueco.

\section{LITERATURA CITADA}

Alvarez, H. 2010. Termoprotección de las partículas reflectantes en frutales de pepita. Evaluación mediante sensores remotos de infrarrojo térmico. $78 \mathrm{p}$. Tesis. INTA Alto Valle, Università Degli Studi Di Bologna, General Roca, Argentina.

Alvarez H.L., C.M. Di Bella., G.M. Colavita, P. Oricchio, and J. Strachnoy. 2015. Comparative effects of kaolin and calcium carbonate on apple fruit surface temperature and leaf net $\mathrm{CO}_{2}$ assimilation. Journal of Applied Horticulture 17:176-180.

Behmer, S.N., A.P. Di Prinzio, C. Mignone, J.C. Magdalena, y A. Stular 2014. Mitigación del asoleado en frutos. Calidad de aplicación de pantallas solares. . Libro de resúmenes, XXXVII Congreso Argentino de Horticultura. № 323, p. 88. ASAHO (Asociacion Argentina de Horticultura), Mendoza, Argentina.

Braekman, P., D. Foqué, W. Messens, M.C. Van Labeke, J.C. Pieters, and D. Nuyttens. 2010. Effect of spray application technique on spray deposition in greenhouse strawberries and tomatoes. Pest Management Science 66(2):203-212.

Chabbal, M.D., A.B. Piccoli, G.C. Martínez, M.M. Avanza, S.M. Mazza, y V.A. Rodríguez. 2014. Aplicaciones de caolín para el control del golpe de sol en mandarino 'Okitsu'. Cultivos Tropicales 35(1):50-56. La Habana, Cuba. ISSN 0258-5936.
Colavita, G.M., V. Blackhall, and S. Valdez. 2011. Effect of kaolin particle films on the temperature and solar injury of pear fruits. Proceedings 11th International Pear Symposium. E. Sánchez et al. (eds.) ISHS. Acta Hort. 909:609-616.

Di Prinzio, A.P., S.N. Behmer, G.L. Striebeck, G. Colavita, y J.C. Magdalena. 2009. Aplicación de una pantalla solar en frutales con un barral vertical. Evaluación de la cobertura en frutos. Libro de Resumenes. XXXII Congreso Argentino de Horticultura. FR. T12, Pág. 219. ASAHO (Asociacion Argentina de Horticultura), Salta, Argentina.

Di Rienzo, J.A., F. Casanoves, M.G. Balzarini, L. Gonzalez, M. Tablada, y C.W. Robledo. 2008. InfoStat, versión 2008. Grupo InfoStat, FCA, Universidad Nacional de Córdoba, Argentina.

Foqué D., and D. Nuyttens. 2011. Effect of nozzle type and configuration on spray deposition in ivy pot plants. Pest Manage. Sci. 67:199-208.

Foqué Dieter, J.G. Pieters, and D. Nuyttens. 2014. Effect of spray angle and spray volume on deposition of a medium droplet spray with air support in ivy pot plants. Pest Management Science 70(3):427-439.

Glenn, M. 2012. The mechanisms of plant stress mitigation by kaolin-based particle films and applications in horticultural and agricultural crops. HortScience 47(6):710-711.

Hellebrand, H.J., H. Beuche, M. Linke, B. Herold, and M. Geyer, 2001. Chances and shortcomings of thermal imaging in the evaluation of horticultural products. International Conference "Physical Methods in Agriculture - Approach to Precision and Quality". Prague, 27-30 August 2001. Proceedings. p. 112-117.

Nuyttens, D., M. De Schampheleire, P. Verboven, E. Brusselman, and D. Dekeyser. 2009. droplet size and velocity characteristics of agricultural sprays. Transactions of the ASABE 52(5):1471-1480.

Onorato, A., y O. Tesouro. 2006. Pulverizaciones agrícolas terrestres. Ediciones INTA (Instituto Nacional de Tecnología Agropecuaria), Buenos Aires, Argentina. 168 p.

Racsko, J., y L.E. Schrader. 2012. Sunburn of apple fruit: historical background, recent advances and future perspectives. Crit. Rev. Plant Sci. 31:455-504.

Raffo Benegas, M. D. y A. Rodríguez. 2007. Factores que afectan el porcentaje de fruta asoleada en manzanos cv. Fuji en el Alto Valle de Río Negro y Neuquén. RIA 36(1):131-146. 